\title{
Transparency in the International Journal of Medical Students
}

\author{
Francisco J. Bonilla-Escobar, ${ }^{1}$ Aisha K. Gharaibeh, ${ }^{2}$ Stuart J. Mires, ${ }^{3}$ Hulegar A. Abhishek. ${ }^{4}$
}

\begin{abstract}
"Science isn't about authority or white coats; it's about following a method. That method is built on core principles: precision and transparency; being clear about your methods; being honest about your results; and drawing a clear line between the results, on the one hand, and your judgment calls about how those results support a hypothesis."
\end{abstract}

Ben Goldacre.

Noted emerging trends in the medical publication world, transparency and best practice in scholarly publishing have recently gained special emphasis. Publication ethics has rapidly grown, especially in medicine, and the need for transparent reporting of research studies is being demanded. Through withholding negative results or falsifying data we endanger not only the reputation of a field, but the evidence base for life-changing decisions that will ultimately affect patients and the health of individuals and society.

Within the scientific community, research results - both negative and positive - will influence evidence for decision making. In Journals, a lack of legitimate internal editorial process and transparency can lead to publication bias. Systematic reviews on publication bias have clearly demonstrated that studies estimated to have publication bias had higher chance of acceptance and were published in higher impact journals.

The International Journal of Medical Students (IJMS) essentially adheres to a transparent editorial process. For example, the guidelines for authors adopted by the IJMS were developed based on the recommendations and guidelines of well-known bodies for medical journal editors, such as the International Committee of Medical Journal Editors (ICMJE), the Committee on Publication Ethics (COPE) and Statements such as the STRO$\mathrm{BE}$ (STrengthening the Reporting of OBservational studies in Epidemiology) [http://www.strobe-statement.org], AGREE (The Appraisal of Guidelines for Research and Evaluation) [http:// www.agreetrust.org/resource-centre/practice-guidelines/], CONSORT (CONsolidated Standards of Reporting Trials) [http:// www.consort-statement.org/consort-statement/], PRISMA (Transparent Reporting of Systemic Reviews and Meta-Analyses) [http://www.prisma-statement.org/] and STARD (STAndards for the Reporting of Diagnostic accuracy studies) [http://www. stard-statement.org/].

Further, the IJMS has improved the submission system and currently applied the Open Journal System (0JS) platform. The 0JS is a validated and well-known platform for journal management, submissions and editorial processing developed by the Public Knowledge Project (http://pkp.sfu.ca/ojs/ [Access: 0129 2014]). It promotes and improves open access research. With this system now in place, IJMS authors and readers will find an easy and step by step process to submit their manuscripts and will have an improved experience throughout the editorial process. Furthermore, as the oJS is a worldwide platform, authors will gain experience in a submission process common in publishing and beneficial to their future careers.

The IJMS has recently required a transparency statement for its authors along with the other requirements. These requirements are: (1) a Cover letter: which should state mainly the novelty, interest and relevance of the work; (2) the Declaration of Conflict of Interest: which should report to the editors and readers any conflicts of interest related to the study; (3) The IJMS Author's Signature Form: in which each author agrees on points required by the IJMS to ensure the integrity of the work produced and state the contributions of each author, provided with the signature and identification card for each author; (4) Template for manuscripts: which outlines the layout of each manuscript type; and (5) Informed consent form for case reports: to avoid any ethical violation for the patient whose case was reported.

The transparency statement that is included for IJMS authors is the following:

The lead author affirms that this manuscript is an honest, accurate, and transparent account of the study being reported; that no important aspects of the study have been omitted; and that any discrepancies from the study as planned (and, if relevant, registered) have been explained. ${ }^{2}$

The inclusion of a transparency statement to be signed or accepted by authors is considered an important recent trend for scientific journals. The COPE, the Directory of Open Access Journals (DOAJ), the Open Access Scholarly Publishers Association (OASPA), and the World Association of Medical Editors (WAME) have collaborated to develop the Principles of Transparency and Best Practice in Scholarly Publishing to differentiate non-legitimate from legitimate publishers. The IJMS fully embraces these principles through the following:

1- Peer review process: for original articles, short communications, reviews and case reports article types; 2- Governing Body and, 3-Editorial team/contact information: the Editorial Board structure along with the ancillary jobs names with their contact information are clearly identified on the IJMS website; 4- Author fees: it is clearly stated that the IJMS does not request any kind of fees; 5- Copyright and licensing information are clearly described on the website; 6 - Identification of and dealing with allegations of research misconduct: the IJMS guidelines for authors draws attention to the avoidance of research misconduct, the editors and reviewers of IJMS are required to identify any

${ }^{1}$ Editor in Chief IJMS. Cisalva Institute, University of Valle, Cali, Colombia.

${ }^{2}$ Scientific Editor IJMS. Jordan University of Science and Technology, Ar Ramtha, Jordan.

${ }^{3}$ Student Editor IJMS. University of Oxford, Oxford, England, UK.

${ }^{4}$ Associate Editor of Original Articles IJMS. Bangalore Medical College and Research Institute, Bangalore, India.

Correspondence:

Francisco J. Bonilla-Escobar, MD, MSc(c)

Address: Cisalva Institute, University of Valle, Cali, Colombia

Email: editor.in.chief@ijms.info 


\section{Editorial}

plagiarism or fabrication in the IJMS Manuscript Evaluation and the Peer Reviews Evaluation Form, respectively. Those two internal evaluation forms were constructed by the IJMS Executive Committee in order for the manuscript editors and reviewers to check the value of submissions to scientific literature; $7-0 \mathrm{w}$ nership and management: stated on the website; 8 - Web site: www.ijms.info; 9- Name of Journal: the International Journal of Medical Students, a unique name and descriptive of the journal mission and vision; 10- Conflicts of interest: adopted from the ICMJE and clearly stated in the instructions for authors; 11- Access: clearly defined as online and open to all readers without subscription fees; 12- Revenue sources; 13- Advertising; 14- Publishing schedule: as tri-annual; 15-Archiving; 16- Direct marketing.

Additional details on each point can be accessed through the WAME website (Available from: http://www.wame.org/resources/principles-of-transparency-and-best-practice-in-scholarly-publishing. Updated 2013 Dec 19, Cited 2014 Jan 31). All of the principles stated are clearly evident on the IJMS website.

Authors do not hold the sole responsibility for publications. Editorial teams and editors complement authors and must follow principles of transparency to guarantee best practice in editorial processing. Thus, the IJMS supports the principles promoted by the EQUATOR (Enhancing the QUAlity and Transparency of health Research) Network (www.equator-network.org [Access: 0129 2014]).

During the editorial process of the IJMS, editors consult the EQUATOR reporting guidelines as part of evaluating the manuscript at hand and request authors adhere to them. Although editors often say that it is difficult to ensure compliance with these reporting guidelines; the IJMS is a unique journal aimed at medical students who should be a primary target for training in good research and publication practice. We hope to establish good habits as researchers and physicians at the beginning of their scientific journeys.

Please feel free to contact our Editorial Team with any questions or discussion related to this topic. Transparency is a central goal for the IJMS, and is key in our continued drive for improvement. The IJMS was created by medical students for medical students and continues to grow to be the most important means of scientific publication for medical students around the world. Through implementing transparency principles we can educate and inspire a generation about the necessity of these approaches to advance the scientific community.

\section{References}

1. Dubben HH, Beck-Bornholdt HP. Systematic review of publication bias in studies on publication bias. BMJ. 2005;331(7514):433-4.

2. Altman DG, Moher D. Declaration of transparency for each research article. An antidote to inadequate reporting of research. BMJ 2013;347:f4796.

\section{Acknowledgments}

None.

Conflict of Interest Statement at Funding

The authors have no funding, financial relationships or conflicts of interest to disclose.

Cite as:

Bonilla-Escobar FJ, Charaibeh A, Mires SJ, Abhishek HA. Transparency in the International Journal of Medical Students. Int J Med Students. 2014;2(1):6-7. 